\title{
The Color Pile: Equitable Self-Expression Through Color and Abstraction
}

\author{
Kathryn de Laszlo \\ Visual literacy and visual resources educator, USA
}

\begin{abstract}
The Color Pile is a visual tool transported from the author's art-student context, and builds on the teaching model of Connie Smith Siegel and the Color Contrast work of Johannes Itten. As re-positioned, it offers a novel path to eliciting student narratives and point of view in language-dependent learning settings. Can this playful exercise support the clear articulation of complex ideas and help generate descriptive language? The Color Pile process moves from prompt to reflection to abstract visual composition, and resolves in a verbal, written or drawn reflection. Color and abstraction may help students gain access to their full capacities for complex thought and self-expression. Could this approach provide differently equitable support for student-produced narratives and descriptive language than is afforded by viewing representational imagery? Direct observations of middle school students using the Color Pile suggest the method could be meaningful to a diverse audience of teachers and learners. Its usefulness in a broad spectrum of language-oriented learning settings is considered.
\end{abstract}

Keywords: Multimodal literacy, personal narrative, culturally responsive teaching, lived experience, expressive color

\section{The Color Pile \& Process Described}

Imagine a field of about 50-100 separate color swatches of all hues, lights and darks, and various degrees of saturation spread out in a random arrangement on a large white sheet of paper (24" x 36" or larger). This is the Color Pile as inspired by the work of Johannes Itten (Itten \& Birren, 2001) and developed by Connie Smith Siegel (Smith Siegel, 2008). The square and rectangular swatches range in size from a few inches to a half of a letter-sized sheet of paper. They are arranged loosely and many color swatches overlap. Care is taken to distribute repeated hues, light and dark colors, and bright and subdued tones. To the casual observer, the arrangement looks arbitrary. The ratio of swatches to students is ideally 10:1 or greater, so that choice of colors is always possible.

Before direct interaction with the Color Pile begins, the teacher/facilitator provides an appropriate visual, written, or spoken prompt for the given community of learners. The prompt may be keyed into specific lesson plans, or it may act as a stand-alone experience. After being given the verbal prompt, the participants are asked to quietly reflect on it for a few moments. Closing their eyes or gazing at their own white workspace is helpful during this visualization. After this reflection, they are free to make selections of roughly six to twenty swatches at their own discretion and arrange them on their oversized white paper, holding their internally visualized response to the prompt in their thoughts as they make their color selections. Their own white paper serves as a canvas for composing. Nothing is ever glued down. The participant's own white paper should accommodate all of their swatch selections, allowing for ample white (negative) space.

The Color Pile builds a community of empathic observers and listeners as each participant shares a verbal narrative response to the prompt and the color interaction, their process, and the meanings they have ascribed to the various color swatches. Finally, time allowing, there is an invitation to draw or write a reflective response to the generated color composition. The entirety of the Color Pile experience can be fit into a one-hour time frame, often allowing for two prompts.

Teaching and Facilitation Contexts

After engaging with the Color Pile Process as a student of Connie Smith Seigel, I directly supported the Color Pile process in four contexts: among adult art students as a peer and student, with middle school 
students as a visual arts teacher, in a curriculum aimed at closing achievement gaps, and with dual immersion teachers. Most recently I have worked directly with middle school students and humanities teachers as well as teachers of English as an additional language.

In order to conform to the budget and the time frame allocated for my middle school visual art students, I had to develop a curriculum that utilized inexpensive portable materials which were compact, tidy and didn't require a lot of technique, skills-acquisition or experience. Colored card stock cut into random sizes, inexpensive pastels and a prompt comprised an affordable kit that could be widely used with a large number of students. In this setting, the Color Pile was aligned with a two-part prompt. Initially, they were asked to use language to write or speak about an invented country in all its dimensions of geography, climate, population, culture, etc. Once they developed a robust description of the country, they used the Color Pile to select four to seven colors that they felt were symbolically appropriate. These colors would be the basis for a flag design. The Flag identity project utilized the Color Pile in a much more prescriptive way, nested in a visual arts curriculum, but it taught me a great deal about the potential for this color and composition work to serve as a platform for identity and constructed narratives.

The Flag identity project was an exercise intended to heighten engagement and provide a sense of empowerment. It served to introduce formal Elements of Art, and aspects of design and symbolism. What surfaced was the readiness of students to form attachments to these abstract colorful visual expressions of a formed place-identity. It was the impetus for re-purposing the Color Pile process into language-oriented contexts and academic classrooms where I felt others (both teachers and students) would benefit. I believed this playful non-prescriptive approach to self-expression and agency would be of even greater value in these settings than in our visual arts context. I had seen the impact it had on L2 students' willingness to describe their imagined worlds and I wanted to carry this into contexts where they were actively struggling to communicate.

\section{The Nature of the Prompt}

Lived experience should be invoked by the prompt in order to access the equitable and empathic dimensions of the Color Pile. This may be most important when the prompt references an integral part of curriculum studies or lesson plans which might include historical events, situations, figures, or narratives in literature, art or film etc. (Hammond, 2014). The prompt may involve people, places, time, objects, sound, environments or situations. In other words, there is a great deal of flexibility and choice placed in the hands of the teacher/facilitator.

As a direct outcome of invoking the lived or imagined experience of the participants, the facilitator encourages memories, visualizations, aspirations and personal perceptions to surface. In practice, it appears to be important to introduce a prompt that is open to multiple interpretations and not too singular in its cultural or linguistic contexts or content. An example might be to reflect on a time when you were able to do something of importance to you for the first time. We might ask, "What did it feel like? Imagine your surroundings in the moment, think about your mood, the sounds, the time of day..." Just as the optical excitement of the colors acts on the participants' senses, the prompt should set the stage to allow for subjectivity and felt experience as valid components of the participants' responses. A marker of success will be the student's own ability to trust their instincts of choice, composition, and expression. The facilitator's task is to support these intuitive responses at every stage of the guided exercise. As they venture into this abstract field of color, focusing on the subjective qualities of individual experience, the participants may find a freedom of expression and a sense of agency. In her own teaching, Connie Smith Siegel (Smith Siegel, 2008) utilized the framework of Johannes Itten's color contrasts (Itten \& Birren, 1970) and applied them toward clearly seeing the express nature of each student through their art. She modelled the potential for visual abstraction in the form of color to act as a reflection of the core sensibilities of the participant, and a path to celebrating the value, and values of the individual in a group setting.

\section{Re-arranging Colors to Locate Expressive Identities}

The flexibility in the color selection and composition process suggests and informs a fluid, unencumbered, and intuitive process. Color has powerful associations with culture and identity, yet the Color Pile explorations I have participated in or observed seem to enable participants to engage in a process of reassignment of meaning. For example, "favorite" colors appear to lose relevance as colors are selected to 
align with a participant's internal response to the prompt. It is important to be watchful for settings with stronger cultural color associations, wherein this transposition of different or new associations could be more challenging. The shifting between the visual medium of color and speaking, may serve as a form of translanguaging, (Garcia, 2009) or enabling movement between cultures, identities and languages in a fluid way (Lewis et al., 2012). The actions of shifting the colors, layering them and re-orienting them on the page is a metaphor for, and a concrete visual expression of the concept of fluid selves, and multiple selves (Henry, 2017).

The participants I have observed work steadily and methodically. They make deliberate selections, and do not appear rushed or hesitant in their body language. They rearrange and layer the color swatches instinctively. When they get close to arriving at an arrangement, their activity will naturally slow down. At this point, I like to suggest that the parent color pile is still available to them. As they shift into arranging their swatches on their separate white page they do so freely, and yet they move steadily toward a sense of arrival - they rarely seem to second-guess their choices once they have arrived at this place. The sense of agency afforded by the invitation to invent and realize their own language through color appears to promote confidence and decisiveness. The facilitator's role is to keep the participant circling back to their internal response to the prompt, and wholly trusting their intuition. Smith Siegel (2008) describes the sense of landing in this way: "As you choose color and draw from your inner sensation you come home to your original essence. And when you come home you can do nothing wrong" (p. 58).

\section{Student Experiences Observed}

I recently facilitated student experiences with the Color Pile in person with two students at a time, ages eight to ten. Each pair of students were also siblings, and in one instance, twins. Some prompts were derived directly from sensory experience relating to memory or everyday experience. Other prompts were keyed into the work or readings the students were concurrently assigned for their online language-arts classes. I facilitated these Color Pile explorations during school closures mandated by the COVID-19 pandemic.

\section{Emmanuel \& Francisco}

Emmanuel (pseudonym) has been invited to engage with the Color Pile for the first time. He knows I am going to invite him to try an activity that involves color and his recent experiences. As he encounters a pile of colored card stock parallelograms on the floor in a random-looking pile he says, confidently, "This is my kind of thing. This is going to work for me."

The first prompt was to stand outside and experience a clear day after persistent wildfires affected the region's air quality. The two brothers, Emmanuel and Francisco (pseudonym), stand outside with their eyes closed to sense the whole feeling of this day. As the facilitator, I suggest they focus on the sensation of the air on their face and arms, and the sounds around them. Afterward, they are asked to sit quietly and close their eyes or gaze at their white paper before selecting from the pile (Figure 1). As soon as they are ready, they are invited to make their selections from the pile. Their movements are full of focus and intention.

In response to the first prompt Emmanuel appears very selective as he steadily pulls swatches from the pile (Figure 2). Later, as he reflects on the process he expresses that he was drawn to a wide range of colors.

I don't know really what to pick, sometimes I feel like l'm a rainbow. All the colors at once. So this can be one big rainbow, every color. And then in the center, the last color. All of them mixed together, black, white and a little bit of grey. And I made them look like they're blocks separated until they go, all of them swirl around, all of them are right now in the middle. They all come together. It kinda looks like a vortex (Figure 3).

After generating the color composition, Emmanuel is given an option to write or draw from it. He chooses to draw. The students use oil pastel for its immediacy, portability, and for access to more saturated colors than colored pencil. The resulting image looks like a loose ball of yarn that loops around an open center of white on the page. But during the exercise this is just the sort of visual description I would refrain from verbalizing. It will be left to the participants to mediate their visual constructions with their own language. At 
the vortex's center is a series of drawn loops in black and many colors, as if the pastel lines were running around a track. The parallelograms that formed the image are softened here and expressed by Emmanuel's circular motion on the page as he draws these overlapping concentric ovals. I describe it visually solely for the purpose of the reader making sense of his description since the drawing is not pictured.

\section{Figure 1}

Emmanuel reflecting on the prompt

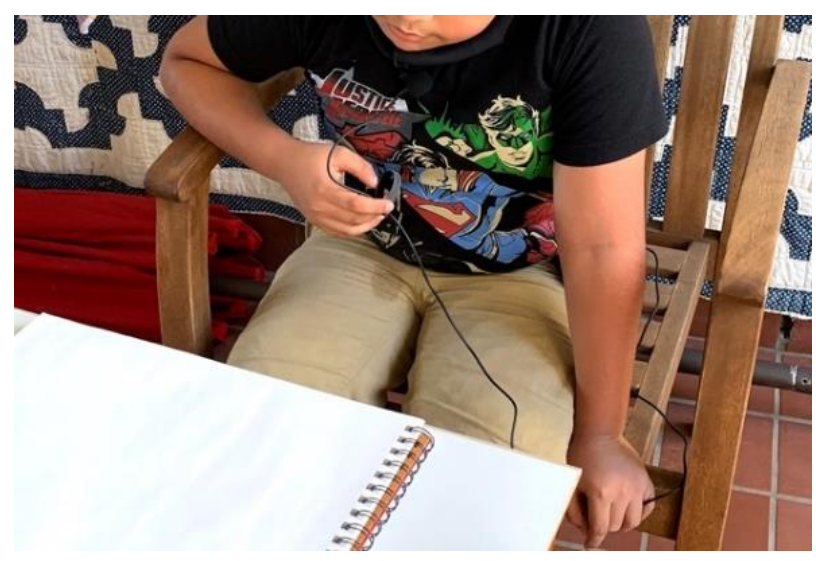

Figure 3

Emmanuel's Vortex composition

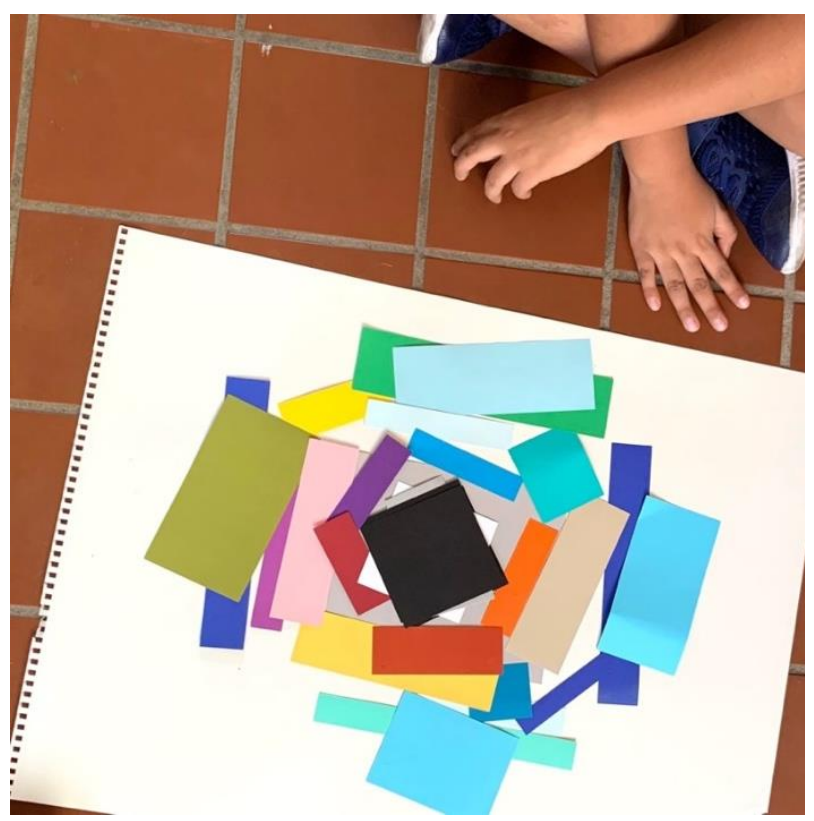

Figure 2

Emmanuel and Francisco selecting from the Color Pile

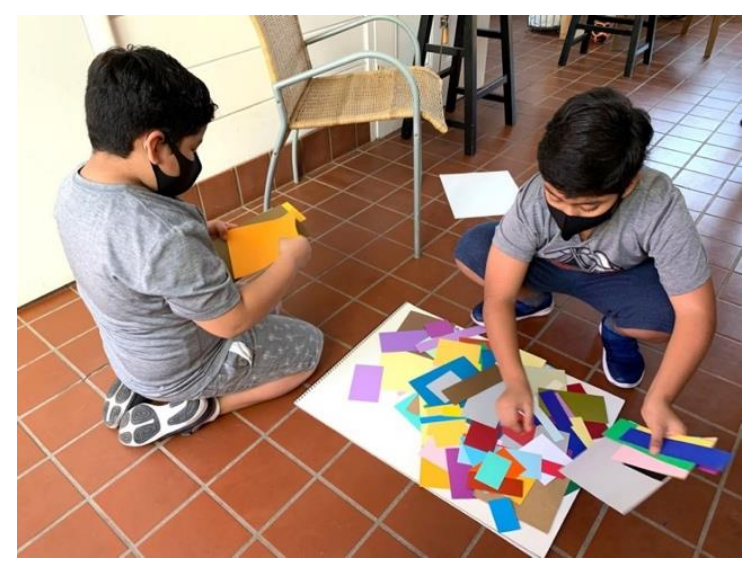

Figure 4

Francisco's Time and Days composition

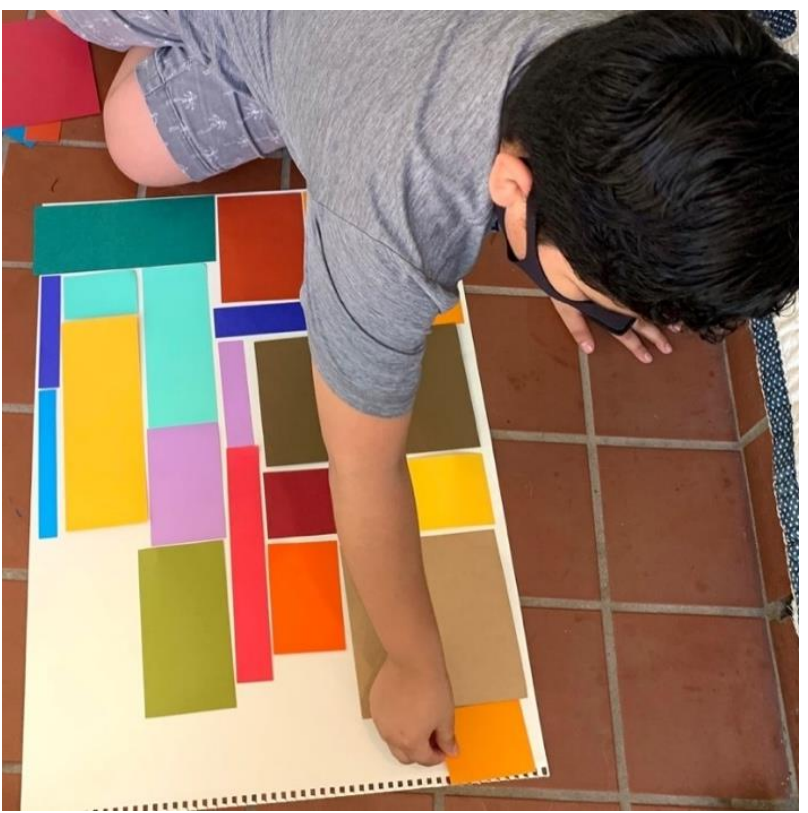

You know how that is squared? (referencing his composition) I decided instead of making it squared, why not just both? If that's one version of what's inside of me, and this is another one, well, so be it. My imagination can go through many forms. A lot, actually. But now it's instead of just being black, it's showing what it actually 
is. But right now, on its outer look it used to look like that now it's turned into a new thing and it looks like thiswhole rainbows. Instead of just being one color.

Emmanuel associated the composition with his felt experience using the Color Pile and identified directly with his rainbow vortex. Notably, he shows[s], "what it actually is", suggesting that the drawing is a concrete expression of the rainbow, and a more exacting expression of his idea. Implicitly, the color composition is a manifestation of one of the many forms his "imagination can go through."

This actually reminds me of something l've drawn before. One time I made with, actually, it took me a long time to get this correct detail but I made a galaxy looking whirlpool. And I made it look like this, And I don't know how to use an oil pastel but I do it more better in paint. And I decided, why not that just be what represents me? And that should just be me. So if anyone wants to know who I am inside, I guess that's really the best thing to represent me.

When Emmanuel is given an open-ended prompt to consider something he was studying in school which he could also attach feeling to, he describes a school field trip to the local creek to observe the salmon spawning. The resulting composition is a representation of a fish (Figure 5). In using the color swatches in an illustrative way, he can't help but consider what the composition looks like-he had shifted out of the abstract mode even before he began the composition. After reflecting on the color swatch composition, Emmanuel draws a representational fish.

\section{Figure 5}

Emmanuel's Salmon with water and pollution

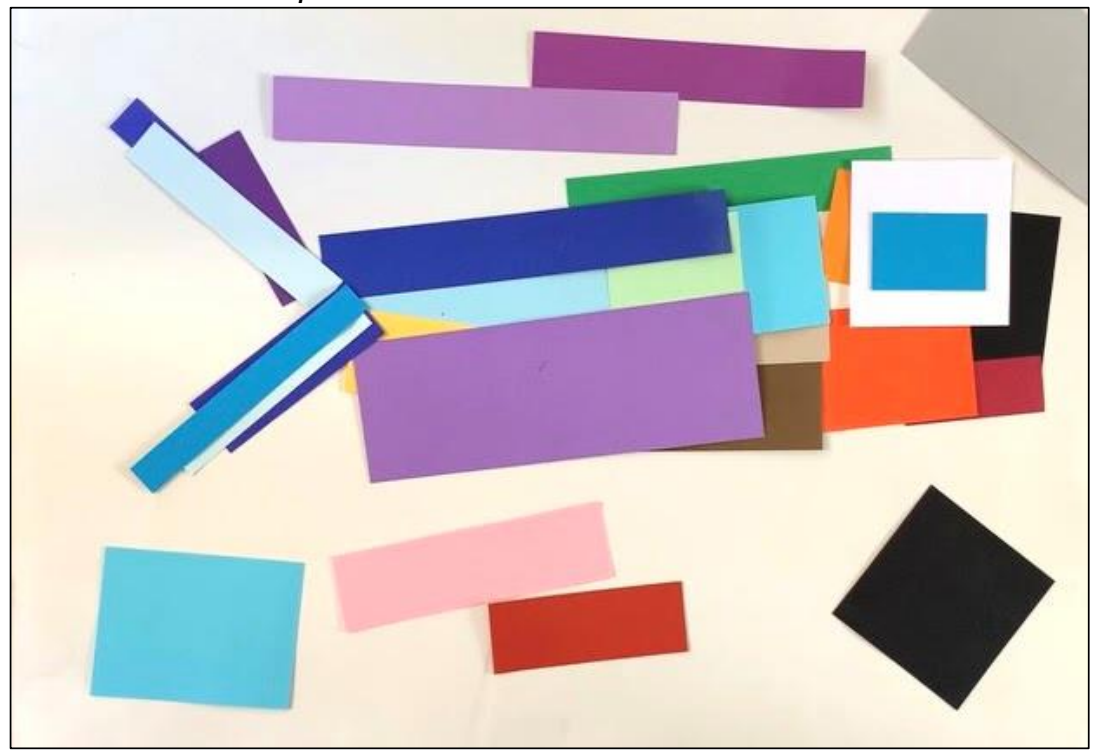

I already had this in my brain already. I had this in my vision already. Like, without even a warning my brain already confirmed to me what I'm gonna make. And the exact colors and exact shapes actually that I was gonna need. So I chose the black and red for the snout and the orange to be the neck...or I think it's supposed to be the neck? I'm not sure if fishes do have a neck, but I do know they have gills. The thin strips are actually it's gushing through the water. But the bigger things that are black and grey are also the pollution. So we wanna make sure they get rid of that so the salmon can go live freely without any pollution in the river. So they can go lay eggs and have babies.

When asked to reflect on a conflict between characters from a story he had read in school, Emmanuel creates a very simple ordered composition. He assigns specific meaning to the swatch's placement by ascribing them the roles of both characters and feelings. This time, after making the drawing, he decides to add text describing both the situation, and the way the composition represents it. 
What I think these colors are is the conflict between the characters in the story. The green would be the largest. This would be his friends in the story. His friends make some decisions he feels guilty about. And this is where the guilt comes in. I chose black because I think that would be best to represent it. This is the solution, so I can say it's stairs. So, I think it's all the events leading up to the solution. Many events going up into the end. Emotions and events (Figure 6).

\section{Figure 6}

Emmanuel's conflict and solution: a response to a work of fiction

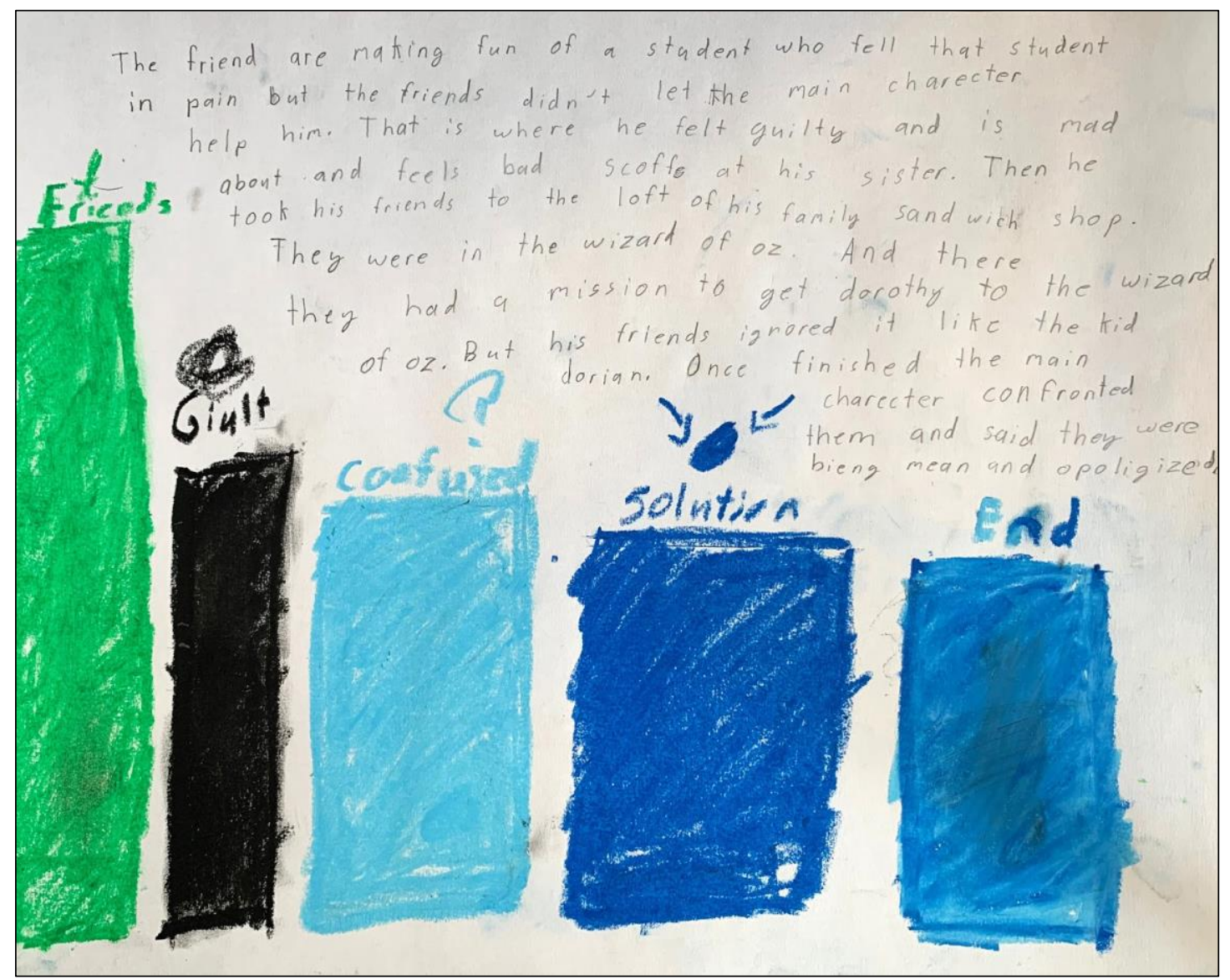

Francisco was given a prompt to reflect on his experience of the day. He created a structured, map-like arrangement of the swatches (Figure 4). His brother, Emmanuel, comments that "it looks like a city." Here, Francisco describes his composition:

Technically the big orange piece is when you really have fun in the day. And the red piece is when you feel like you can do more. And the green piece makes me feel like it's a really long day. And the yellow piece makes me think of the year. And the tiny little line pieces, those make me think of like, the time where it's so happy, like it can go on forever. And this tiny little piece makes me think of it's a nice day outside, and it's really shiny. There's a lot of colors. Today feels like it's the orange. The orange? Because today's a Wednesday, so that means-Wednesday on Zoom-that means it's only gonna be 30 minutes. And I'm almost done with all my homework. Like, technically means I have more free time.

\section{Ben \& Ava}

Ben(pseudonym) is 11. He arrives with his twin sister and their mother. I am meeting the kids for the first time. They are quiet and appear cautiously curious. With gently expressed enthusiasm, they state their 
readiness to try it out. The prompt is to simply sit quietly and imagine an experience they had that day, including the feelings, sounds, smells, and sights associated with the experience.

When it's time to make their selections, they work at a moderate pace, in a thoughtful rhythm. What Ben composes is an orderly arrangement. It has a grid-like "mapping" look similar to Francisco's image. Ben uses the swatches to describe a familiar physical space that he experienced that day, carefully overlaying some swatches to reduce the visible size of others as he works. When asked if he wants to write or draw in response to his composition, he chooses to draw. When it is time to reflect on the color exploration, his spoken narrative is over 270 words, with some intertwined (additional) queries from me (K). Here is an excerpt from Ben's description of his Color Pile arrangement:

\section{$\mathrm{K}:$ Tell me about this.}

Ben: Well this is a place we went whenever, well usually when we go on walks we walk through this place. It's an alley. Here's the fences, they aren't really to scale, this is the alley like the gravel, the road at the other end and this is just the sky and the sun. This is one of the people on the way who owns a yellow Mini Cooper in their driveway. And then these are the fences one person has this huge yard with a bunch of trees. Here are the trees they rise above the fence really high. This building is the school district's maintenance building. It's across the street from the alley, we usually turn, and then here's the sun. I couldn't find any squares that were small enough. And this is just the sky down low, uh yeah, and then it got bluer up. I guess you can call these the clouds but I couldn't find any pieces that would fit there. Usually the sky kind of gets into a deeper blue as you look up. That's why I layered the blues and the sun was relatively hot when we took that walk. And here's the... so some people on this alleyway have this yellow Mini Cooper that I always notice, it's always parked, here it is.

$\mathrm{K}$ : So you were outside, and was there a feeling attached to being outside that you feel like is also in this?

Ben: Well like we're walking the dog and also getting some exercise. It was... fun, I like going outside. I always get excited so does the dog, that we're gonna get outside. So, like...I finish early with my schoolwork...And then I just get bored and I just stand around the house waiting for us to go somewhere so I get excited when we go on walks.

Ben describes selecting his colors:

Picking the colors was pretty easy. All I really needed to do was just figure out the scale of how big everything should be and then like how long the pieces and stuff should be so that they fit in proportion to the real alley.

Ava (pseudonym)'s composition was very dynamic, with a strong presence of contrast of hue (Itten \& Birren, 1970 ) and a visually busy arrangement of many swatches on the page.

Ava's responses are in direct contrast to Ben's. Her memorable experience of the day was sitting in the house reading, and her composition was an expressive description of reading. To refer to the colors and drawing does not generate as much descriptive language for her. For Ava, the creation of a simple but powerful metaphor seems adequate. The pleasure of reading, she explains, is akin to an explosion. Her simple spare words describe a vivid, powerful arrangement of color. When she describes the "explosion" of thoughts in her head, she appears to both surprise herself with her verbal description, and to be pleased, as if she has discovered something exciting and familiar that she wasn't able to previously articulate about her own experience, (prior to making her arrangement). I was left to wonder if she would have arrived at this analogy if someone had simply asked her whether she enjoyed reading.

K: I just want you to be my guide to what you've created here. Just tell me about it in any way you feel comfortable.

Ava: Um... I dunno I was reading and I like to read, I was happy, and so that's sort of what I made.

K: How do you feel that you were drawn to certain colors to express that place?

Ava: It's more like they were bright and random. Like if I was sad they'd be more stripey and dark colors. 
$\mathrm{K}$ : When you look at it, like if you were gonna be the tour guide to what...

Ava: Looks like an explosion of color, like a paint explosion or something.

$\mathrm{K}:$ And when you were reading did it feel like that?

Ava: I really like to read.

$\mathrm{K}$ : So if you were to describe the explosion what kind of explosion would you call it?

Ava: Well I'm not sure, it's not like a bad explosion like a bomb, it's more like just a bunch of colors, like a whirl of colors or something.

\section{A Visual Celebration of Identities}

The simple act of choosing and arranging color swatches seems to present a compact yet powerful opportunity for the student/participant to feel celebrated. The invitation to choose the colors serves as an act of self-affirmation. The student/participants appear to listen closely to their instincts and their sympathetic selves as they compose. The process has a palpable feeling of serenity and grace which has an uplifting impact on the individual and the collective group. This is supported by the relative silence of this parallel activity in a shared space. The support for a prevailing climate of positive engagement is evidenced by the rapt attention and relaxed posture of the participants. The reflective interaction with the colors provides a welcome distance from the omnipresence of language. Imagery, including that which we create ourselves, "provides an alternate way of organizing thought, reality, and self," thus "compensating for the coercive force and structural limitations of language" (Fleckenstein, 1996, p. 920).

\section{Quieting the Prescriptive Language(s) of Art}

The Color Pile's vibrant outcomes consistently suggest experience with visual composition, regardless of the participants' previous exposure to composing with form and color. The non-referential shapes seem to foster an efficient understanding of weight, and rhythm. Although the resulting color swatch arrangements may exhibit notably affective, balanced, and confident compositions, they are not intended to be analyzed using art and design rubrics. The vocabularies of formal visual analysis and the modes of critique and assessment inherited from art-practice and art-historical domains are not an asset to an equity-oriented version of the Color Pile process. Instead, we seek to elicit language and narratives of the self. To position these participant-generated arrangements as a lesson in the elements of art or principles of design would be to abandon many of the values the process upholds, including the creator's ability to linguistically reinvent symbolic and personal meanings, as they translate their own impressions from the paper into spoken narratives and descriptions.

I do not intend to delimit access to aesthetic or art world values and ways of seeing and understanding (Greene, 2001; Eisner, 2003), but to propose that an actual conflict of objectives and methods will present if we attempt to didactically teach color theory and composition in conjunction with the Color Pile and its outcomes in this context. Smith Seigel's and Itten's allegiance to the intuitive and emotional power of color relationships brought us to this application of the Color Pile, not an analysis of color as an objective optical phenomenon. Nonetheless, the activation of visual literacy skills may be a naturally occurring outcome of the process, and this bears further inquiry. Greene would have the art world's skills of looking be intentionally introduced, but she admits the possibility of a crisis for the viewer as they admit or omit content while viewing visual art (Greene,1994). This may be a cultural crisis as well as a visual one. It is the work of critical visual literacy to address this. Abstraction and creation by the participant circumnavigates this need to edit the seen world through that formal lens. The Color Pile's value is an opportunity to support direct, intuitive expression of thought, feeling and experience through visual interaction with color. It is a luxury in a language-learning or language-based classroom to favor action-based, multimodal engagement, and the power to efficiently and spatially manipulate thoughts and ideas ahead of language (Albers \& Sanders, 2010). Acknowledging this will help us to put aside assessment perspectives and culturallyspecific frameworks, and allow for this compact opportunity to support expression and invention in full measure. 


\section{Beyond Representational Content}

The Color Pile provides opportunities to construct and express new meanings while facilitating the manifestation of students' thoughts, without explicit external cultural references at work. Representational visual content carries cultural meaning, as does the act of viewing it and interpreting it. Most visual content, especially when produced or reproduced as visual information or communication, expresses explicit or implicit orientations toward cultural references and perspectives. Representational imagery might include, but not is limited to still images, film, and representational art with commonly identifiable content. It attempts to mirror the visible or visibly-identifiable world. This realm can also include all forms of realism and surrealism and other reconstructions of tangible content which we seek to verify through visual observation. All of these variations are ripe with specific object-anchors and, particularly with photography and film, they act as placeholders-often suggesting singular truths of a positioned, explicit or implied nature.

Visual Thinking Strategies (VTS,), has long recognized the importance of utilizing visual content that offers multiple interpretations through ambiguity. In other words, VTS stresses the value of ambiguity in image selection to encourage multiple interpretations. Visual content in which the contexts or object anchors are uncertain will inevitably enable participant-viewers to attach a broad spectrum of meanings and enable open-ended interpretations. Abstraction through color leverages a form of "strategic ambiguity" (Bratslavsky et al., 2019). Coupled with placing the participants in the role of the designers, (Albers \& Harste, 2007) this enables a positive relationship to uncertainty. The Color Pile may offer an important foundation for helping participant viewers to be more at ease with ambiguous visual information and multiple meanings.

Working abstractly, the Color Pile supports invented, imagined and subjective truths to convey the power of the imagination. A learning culture able to receive these unique points of view supports a classroom culture of acceptance. The abstract nature of the exercise presents a broad spectrum of possibility from the fantastical and metaphorical-Ava's explosive mindset while reading-to the concrete: Ben's detailed mapping of a neighborhood. Some participants draw on felt experience, while others, like Emmanuel, focus on philosophical, symbolic, or inter-relational understandings. Francisco merges the symbolism of color with the concreteness of a mapping approach, and the whimsical attachment of feelings to specific colors and the days they represent.

In this realm of repositioning color we still have to stay attuned as facilitators to the possibility of strong or fixed color associations for some cultures and individuals. The abstract nature of the process lends itself to open-ended interpretation and fluid, complex thinking and re-valuing. It seems to be particularly wellpositioned for constructing and reflecting on and re-constructing established narratives. This is evident in Emmanuel's development of specific colors to represent individual characters and the abstract concept of guilt (Figure 6).

\section{Visual Thinking Strategies Inspired Viewing Contexts}

Experience with the Color Pile has led me to distinguish it from other ways of collectively looking and verbally describing. It allows for uniquely informed and individuated points of view expressed by each individual. In the VTS model, viewers verbally and collectively share their observations without crosscommentary while looking at an image, a work of art or an object, or engage with and reflect on visually observing another art form, or sensory experience. Collectively, participant viewers form a community in this way, creating a layered collective story derived from the seen world (Housen, 2001; Yenawine, 2013). The facilitator carefully paraphrases each verbal contribution for the group.

The Color Pile is related to VTS, but shifts away from the echoes that often occur and the layered meanings which create opportunities to note sameness and difference of perspectives through looking and speaking. It creates a supportive context for seeing others more clearly and highlights the value of being seen and heard. Like VTS, the Color Pile is built on trust and acceptance of the inviolate nature of what each participant has described. Yet because the viewer is also the maker, we are celebrating a projection of the individual which is closely tied to the person, the ego, and the creation of a sense of self. Each component stage of these related approaches reveal opportunities to construct meaning as participants use different strategies of viewing, speaking and listening (Housen, 2001). 


\section{Choice and Agency}

If the Color Pile can act to advance the possibilities for constructing and expressing meaning for students who feel marginalized, unseen, or unheard, then I would assert that it has inherent value in many teaching and learning contexts. Choice and agency provide a robust climate for engagement, and a palpable sense of empowerment. Access to this already portable and economically accessible practice has been extended to a virtual medium during the COVID-19 pandemic as I created an online interactive version. This further extends access to the Color Pile practice as an agency-oriented visual opportunity (Lindgren et al., 2012). As a form of engagement, it is compact, portable, and transferable between language-based disciplines and additional language contexts. It is not intended to call into question the validity or inevitability of language domains or representational imagery, but to offer an alternate and parallel literacy for students as they make their meanings and understandings manifest in a visual way.

The Color Pile supports the creation of a spontaneous abstract language of color, composition, and scale. This, in turn, appears to be readily translated into verbal language by its maker. The participant is the expert: they are the architect of this symbolic mapping of personal narrative and meaning and they are therefore its most competent (and confident) translator and interpreter. Siegel called engagement with the Color Pile an experiment, highlighting the sense of exploration and discovery in a controlled environment (Smith Siegel, 2008). The student-participant appears to own the process from the first encounter.

Simply acknowledging the literacies of students' diverse home cultures is not equivalent to respecting these cultures in learning settings (Harste, 2001). In order to see students holistically we need opportunities to hear from them directly, and they need the affirmation of hearing themselves speak and experiencing themselves being heard. This enables a filtering out of imposed stories and enables them to compose their own truths (Bell, 2002). Using the architecture of color to build narratives we can give students with tentative relationships to language a visual voice, instead of relegating them to being passengers on someone else's narrative. In this way, we grant them new opportunities to share what they perceive, and know and validate what they see, feel and sense as additional ways of knowing. Writing is not the only way to express the knowable (Eisner, 2003). In turn, they will begin to define the culture and community of their learning environments. The quality of attentiveness is both explicitly and implicitly present in the Color Pile practice. Hammond emphasizes that inattentiveness to the individual sets up a shift in the potential of the brain and nervous system to fully function in a learning environment (Hammond, 2014). The importance of introducing such opportunities is an act of meaningful inclusion for students who are challenged by their access to language competencies or otherwise culturally, racially, economically, or individually marginalized. As Jewitt (2002, p. 244) stresses, "...the official institutional construction of literacy may or may not dovetail with emergent practices in homes and communities." Providing safe spaces to self-express is a matter of interest to the collective body of students in a learning environment as well as each individual.

\section{New Contexts}

The conditions of the pandemic created an impetus for developing an online, interactive version of the Color Pile. This has enabled continued and expanded work with teachers in training and L2 teachers in different parts of the country, and the world, including Japan, Uruguay and Turkey. I have begun to record participant English teachers and language learners interacting in small groups online with the Color Pile process. Formal qualitative research utilizing the Color Pile might shed light on the capacity for abstraction and ambiguity to support multimodal learning and cultural and personal re-interpretations. Where I have recorded participant interactions, I have begun to note patterns of expression and a marked sense of attachment among participants to their expressed meanings and a marked appreciation among participants. The nature of these attachments is worthy of further exploration. Research into the Color Pile's ability to impact student anxiety and potential in relation to language learning is worth exploring (Peng, 2014) , including focusing on the role of anxiety in suppressing the ability to self-express in L2 settings.

\section{Conclusion}

The Color Pile may offer a visual respite from linguistic fatigue, and the anxieties of generating language on the spot. In language-learning, and in language-oriented learning contexts, it can mediate the biases of language proficiency and the over-weighting of dominant cultures. The Color Pile may have a positive influence when and where the conventions of language learning and representational content present as 
de-limiting to disparate perspectives, and ways of seeing and understanding. The Color Pile contains constructed knowledge domains inclusive of language and culture (Harste, 2001), while acting at the juncture of visual, verbal, and lived culture. One approach to advancing literacies of the individual is to advance multimodal access to visual literacies and ways of knowing. Embedding the Color Pile in a lesson plan in a group context can act as a social experience. Perceptions of self and others are brought to bear on the broader contextual and content-based classroom culture. It may help students become more adept at expressing and advancing their unique perspectives. The weaving of color symbolism and metaphor introduces new ways of thinking and making experience visible. Where language literacy and or representational visual content are the dominant modes of communication, the Color Pile may provide an alternative opportunity for expanded student participation, engagement, and equity by interjecting a visual frame of reference. As a practice, it encourages us to put aside classifying and narrowing knowledge domains (Harste). Many perspectives, as defined by lived experience, contribute to a unique cultural, social, and personal context formed by a community of peers through this visual and verbal language of description and translation. As we give voice to these realms, we actively reinvent our own histories by constructing new connections (Housen, 1999). We can draw an analogy between the dynamic nature of color interaction causing one hue to alter our perception of another, and the possibility that one or many individuals in such a group will have the capacity to shift the understandings, the perceptions, (and more robustly, the culture) of others.

\section{References}

Albers, P., \& Harste, J. (2007). The arts, new literacies, and multimodality. English Education, 40(1),6-20

Albers, P., \& Sanders, J. (2010). Literacies, the arts, and multimodality. Urbana, III: National Council of Teachers of English.

Bell, J. (2002). Narrative inquiry: more than just telling stories. TESOL Quarterly, 36(2), 207-213. doi: $10.2307 / 3588331$

Bratslavsky, L., Wright, A., Kritselis, A., \& Luftig, D. (2019). The strategically ambiguous assignment: an approach to promoting critical and creative thinking in visual communication. Journal of Visual Literacy, 38:4, 285-304, doi:10.1080/1051144X.2019.1673999

Fleckenstein, K. (1996). Images, words, and narrative epistemology. College English, 58(8), 914-933. doi: $10.2307 / 378229$

Eisner, E. W. (2003) The Arts and the creation of mind. Language Arts, 80(5), 340-344.

Garcia-Mateus, S., Palmer, K. (2017). Translanguaging Pedagogies for positive identities in two-way dual language bilingual education Journal of Language Identity \& Education doi:10.1080/15348458.2017.1329016

Greene, M. (1994). Postmodernism and the crisis of representation. English Education, 26(4), 206-219.

Greene, M. (2001). Releasing the Imagination: Essays on Arts, Education and Social Change. Teachers College Press.

Lewis, G., Jones, B., \& Baker, C. (2012). Translanguaging: developing its conceptualisation and contextualisation. Educational Research and Evaluation, 18:7, 655-670, doi: $10.1080 / 13803611.2012 .718490$

Hammond, Z. (2015). Culturally Responsive Teaching and the Brain: Promoting Authentic Engagement and Rigor Among Culturally and Linguistically Diverse Students. Thousand Oaks, CA: Corwin. 
Harste, J. (2001). What education as inquiry is and isn't. Critiquing Whole Language and Classroom Inquiry, 1-36.

Henry, A. (2017). Beyond global english:motivation to learn languages in a multicultural world. The Modern Language Journal, Vol. 101(3), (Fall 2017), pp. 548-565. Published by: Wiley on behalf of the National Federation of Modern Language Teachers Associations

Housen, A. (1999). Eye of the beholder: research, theory and practice [Conference presentation]. Aesthetic and art education: A Transdisciplinary approach. Lisbon, Portugal.

Housen, A. (2001). Visual understanding in education. Arts and Learning Research Journal, 17(1).

Itten, J., \& Birren, F. (1970). The Elements of Color: A Treatise on the Color System of Johannes Itten, Based on his Book the Art of Color. New York: Van Nostrand Reinold Co.

Jewitt, C. (2008). Multimodality and literacy in school classrooms. Review of Research in Education, 32, 241-267.

Lindgren, D., Lindgren, R., \& McDaniel, R. (2012). Transforming online learning through narrative and student agency. Journal of Educational Technology \& Society, 15(4), 344-355.

Pavlenko, A. (2002). Narrative study: whose story is it, anyway? TESOL Quarterly, 36(2), 213-218. doi: $10.2307 / 3588332$

Peng, J-E. (2014). L2 motivational self system, attitudes, and affect as predictors of L2 WTC: an Imagined community perspective. Asia-Pacific Edu Res 24, 433-443 (2015). doi: $10.1007 / s 40299-014-0195-0$

Siegel, C. S. (2008). Spirit of Color: A Sensory Meditation Guide to Creative Expression. New York: Watson-Guptill.

Yenawine, P. (2013). Visual Thinking Strategies: Using Art to Deepen Learning Across School Discipline (1st ed.). Harvard Education Press.

\section{APA citation format $\left(7^{\text {th }}\right.$ edition) for this publication:}

De Laszlo, K. (2021). The Color Pile: Equitable Self-Expression Through Color and Abstraction. In J. Lee, S. M. Christensen, S. Beene, X. Chen, and W. Huang (Eds.), Visual literacy in the virtual realm: The book of selected readings 2021 (pp. 1-12). International Visual Literacy Association. https://doi.org/10.52917/ivlatbsr.2021.011 